\title{
Diagnostic Performance of ElastPQ Point Shear Wave Elastography for Hepatic Fibrosis in Hepatitis B and Hepatitis C Patients
}

HAGALAHALLI NAGARAJEGOWDA PRADEEP' ${ }^{1}$ SEETHARAM MAHESH ${ }^{2}$, NAGARAJU K RASHMI ${ }^{3}$, NAGARAJAIAH CHANDANDUR PRADEEPKUMAR ${ }^{4}$, CHAKENAHALLI PUTTARAJU NANJARAJ ${ }^{5}$,

\section{ABSTRACT}

Introduction: Chronic viral hepatitis is a condition of hepatotropic viral infection associated with chronic inflammation, hepatocyte injury and progressive fibrosis. Non-invasive assessment of liver fibrosis in chronic hepatitis $B$ and $C$ patients has been increasing and used instead of liver biopsy.

Aim: To assess the diagnostic performance of ElastPQ Point Shear Wave Elastography (ElastPQ PSWE) with liver biopsy as the gold standard and comparing these results with other serum fibrosis markers namely Aspartate to Platelet Ratio Index (APRI), Fibrosis-4 Score (FIB4).

Materials and Methods: A cross-sectional study was carried out in which 73 patients underwent ElastPQ PSWE and Histopathological Examination (HPE) with METAVIR Scoring system staging. Serum fibrosis indices including APRI and FIB4 values were calculated. The diagnostic performance of ElastPQ PSWE, APRI and FIB4 was evaluated using Area Under Receiver Operating Characteristic (AUROCAUROC) curve analysis, correlations of ElastPQ PSWE, APRI and FIB4 ratio index with histopathological findings (as the reference standard) were determined using Spearman's correlation coefficient.
Results: Study included 73 patients with chronic hepatitis B and hepatitis C, of which $8(10.96 \%)$ were no/early fibrosis (F0F1); 20 (27.40\%) were significant fibrosis (F2); 22 (30.13\%) were severe fibrosis (F3), and 23 (31.51\%) were cirrhosis (F4). There was a significant positive correlation between different stages of liver fibrosis by liver biopsy and liver stiffness detected by ElastPQ PSWE $(r=0.912, p<0.0001)$. ElastPQ PSWE exhibited higher diagnostic accuracy than the APRI and FIB4 for the diagnosis of F0-F1 AUROC $0.980,0.730,0.710$, respectively; F2 AUROC 0.840, 0.662, 0.669 respectively; F3 0.564, 0.528, 0.500 AUROC, respectively; and F4 0.967, 0.783, 0.751 AUROC, respectively. Using AUROC curve, the optimal cut-off of ElastPQ PSWE for stages F0-F1 is $\leq 3.9$ kilopascals (kPa); F2 is $\leq 7.2 \mathrm{kPa}$; $F 3$ is $\geq 7.2 \mathrm{kPa}$ and $F 4$ is $\geq 10 \mathrm{kPa}$.

Conclusion: ElastPQ PSWE is a promising non-invasive method used for the evaluation of liver fibrosis, with high diagnostic performance, high Negative Predictive Value (NPV) and good specificity in evaluation of hepatitis $B$ and hepatitis $C$ patients.

Keywords: Chronic viral hepatitis, Liver fibrosis, Non-invasive tissue stiffness assessment

\section{INTRODUCTION}

The management of chronic hepatitis including the treatment, prognosis and follow-up depends on the stage of hepatic fibrosis [1-3]. Liver biopsy is considered the gold standard reference for staging the hepatic fibrosis. Liver biopsy is an imperfect reference standard with limitations in itself being an invasive technique, sampling variability [4] has intra and inter-observer variability [5] and severe post-procedural complications. This has led to assessment of non-invasive techniques such as serological fibrosis markers and radiological tools including ultrasound and magnetic resonance elastography techniques which can diagnose, stage liver fibrosis with high accuracy and also replace liver biopsy [6]. There are four main methods for non-invasive tissue stiffness-based on assessment of liver fibrosis: Transient elastography, PSWE, TwoDimensional Shear Wave Elastography (2D-SWE), and Magnetic resonance elastography [7]. ElastPQ PSWE technique uses conventional ultrasound machine, it can be cost effective, easy available alternative, without the limitations of gold standard liver biopsy to assess hepatic fibrosis [8,9]. However, not many studies have been published evaluating the performance of ElastPQ PSWE in hepatic fibrosis in chronic viral hepatitis $[10,11]$

Hence, the aim of the study was to evaluate diagnostic performance of ElastPQ PSWE in assessment of liver fibrosis in patients with Hepatitis B and C infections and compare diagnostic accuracies of
ElastPQ PSWE with serum fibrosis markers (APRI, FIB4) by using histological liver biopsy evaluation as reference standard.

\section{MATERIALS AND METHODS}

A cross-sectional study was conducted in Krishna Rajendra Tertiary Care Hospital attached to Mysore Medical College and Research Institute, Mysore, Karnataka, India from the patients who were referred to the Radiology Department from February 2020 to October 2020. The Institutional Ethical Committee (IEC) approval was obtained with reference number EC REG: ECR/134/Inst/KA/2013/RR-19.

Inclusion criteria: Patient's positive for hepatitis $B$ and hepatitis $C$ seromarkers between age group of 18-75 years with acceptance of ElastPQ PSWE, clinical and laboratory examination and liver biopsy were included in the study.

Exclusion criteria: Patients having co-infection with HIV; with history of autoimmune hepatitis or primary biliary cirrhosis; with history of alcohol abuse ( $\geq 40 \mathrm{mg} /$ day); patients with hepatic congestion or bile duct dilation evidenced by ultrasound; patients with contraindications for percutaneous liver biopsy; and those who cannot comply with study protocol were excluded from the study.

All patients were subjected to complete clinical examination including age, gender and Body Mass Index (BMI) and laboratory tests which included Complete Blood Count (CBC), liver function test, Aspartate Aminotransferase (AST) and Alanine Aminotransferase (ALT), hepatitis 
seromarkers for hepatitis C ( $\mathrm{HCV} \mathrm{Ab})$ and hepatitis B (HBsAg) using ELISA technique and HCV RNA using Polymerase Chain Reaction (PCR).

The following biomarker parameters were derived and are as follows: APRI calculated as $\{$ AST (U/L)/upper limit normal/platelets (X10\%/L)\} X100 $[12,13]$. FIB4 calculated as age $(\mathrm{yr}) \mathrm{XAST}(\mathrm{U} / \mathrm{L}) /$ PPlatelets $\left.\left(10^{\%} / \mathrm{L}\right)\right\}$ $X\left\{A L T(U / L)^{1 / 2}\right\}[13]$.

Liver stiffness measurement: After taking informed consent, all patients underwent abdominal ultrasonography; then measurement of hepatic stiffness were done by SWE technique (Elast PQ) using 1-5.0 MHZ high frequency curvilinear transducer (C5-1) on Philips Affiniti 70 ultrasound machine (PHILIPS medical systems, Bothell, WA) with the subject in supine or slight $\left(30^{\circ}\right)$ left lateral decubitus position. Subjects were encouraged for shallow breath hold for a few seconds. The Region Of Interest (ROI) was positioned on B-mode image of the liver in the right lobe of liver (typically segment VII or VIII) about 1.5 to $2 \mathrm{~cm}$ beneath the Glisson capsule, perpendicular to the liver capsule and elastography measurements were obtained. Multiple measurements were made. Median of 10 valid measurements was taken. The values of liver stiffness are expressed in kilopascals (kPa). Reliable examination was considered if a success rate of $60 \%$, an inter-quartile range (variability in the validated measures) $<30 \%$ of the median elasticity [7].

Liver biopsy: All patients underwent ultrasound-assisted percutaneous liver biopsy through an intercostal approach with a 17G Menghini needle; biopsy specimens were fixed in formalin and embedded in paraffin. Assessment of the specimen length and the portal tracts number included was done with the length of $1.2 \mathrm{~cm}$ and portal tracts 6 considered as adequate biopsy. Liver fibrosis stage were recorded using the criteria of the METAVIR scoring systems composed of progressive stages: F0 (normal), F1 (portal fibrosis without septa), F2 (portal fibrosis few fibrotic septae), F3 (portal fibrosis with numerous septae) and F4 (cirrhosis) [14]. Liver fibrosis stages determined using the criteria of the METAVIR scoring system were compared with the measurement of hepatic stiffness done by SWE technique (ElastPQ) and also with the APRI and FIB4 Score.

\section{STATISTICAL ANALYSIS}

Correlation between ElastPQ PSWE and the stages of hepatic fibrosis was estimated using the Spearman's correlation coefficient. The diagnostic performance of ElastPQ PSWE, APRI and FIB4 for hepatic fibrosis was determined in terms of sensitivity, specificity, PPV and NPV, as well as likelihood ratio by AUROC curves. The optimal cut-off values between the stages of hepatic fibrosis were determined at the maximised sensitivity and specificity. Analysis of variance (ANOVA) was used to find the significance of study parameters between three or more groups of patients. The $p$ value of $0.05<p<0.10$ is considered significant; $p$ value: $0.01<p \leq 0.05$ was considered moderately significant and $p$ value: $p \leq 0.01$ is considered strongly significant with a 95\%confidence interval. The Statistical software Statistical Package for the Social Sciences (SPSS) 22.0, and $R$ environment version 3.2.2 were used.

\section{RESULTS}

Study included 73 patients with chronic hepatitis B and C. There were 59 males and 14 females included in the study. Their ages ranged between 26 and 77 years with the mean age was $53.12 \pm 11.63$. The mean BMI was $25.51 \pm 3.28 \mathrm{~kg} / \mathrm{m}^{2}$ [Table/Fig-1]

Histological results: The patients were classified according to METAVIR scoring systems, 8 of them were (10.96\%) were F0-F1; 20 (27.4\%) were F2; 22 (30.13\%) were F3, and 23 (31.51\%) were F4. The mean hepatic stiffness measured by ElastPQ PSWE for each fibrosis stage is summarised in [Table/Fig-2]. The difference between several groups were statistically significant (ANOVA=128.234, $p<0.001$ ).

There was a significant correlation between ElastPQ PSWE and liver biopsy, correlation between APRI and liver biopsy, correlation between FIB4 and liver biopsy [Table/Fig-3].

\begin{tabular}{|c|c|c|}
\hline Characteristics & Hepatitis $B$ and Hepatitis C (Mean \pm SD) & $\mathrm{p}$-value \\
\hline Age (Years) & $53.12 \pm 11.63$ & $<0.001^{\star \star}$ \\
\hline Male-n (\%) & 59 (80.8\%) & $0.054^{+}$ \\
\hline BMI $\left(\mathrm{kg} / \mathrm{m}^{2}\right)$ & $25.51 \pm 3.28$ & $<0.001^{\star \star}$ \\
\hline Platelet count $\left(10^{9} / \mathrm{L}\right)$ & $164.34 \pm 74.3$ & 0.164 \\
\hline ALT (U/L) & $81.96 \pm 42.4$ & $<0.001^{\star \star}$ \\
\hline AST (U/L) & $77.36 \pm 32.96$ & $<0.001^{\star \star}$ \\
\hline \multicolumn{3}{|c|}{ 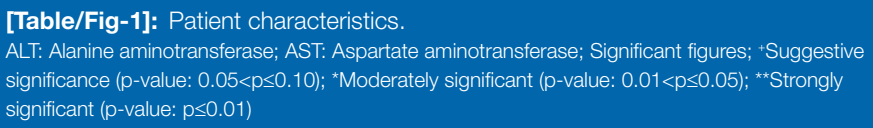 } \\
\hline
\end{tabular}

\begin{tabular}{|l|c|c|c|}
\hline Stages & No. of patients (n) & Mean (kP/sec) & SD \\
\hline Mild/No fibrosis (FO-F1) & 08 & 3.8000 & 1.6920 \\
\hline Significant fibrosis (F2) & 20 & 6.5900 & 1.0167 \\
\hline Advanced fibrosis (F3) & 22 & 9.2955 & 0.2400 \\
\hline Cirrhosis (F4) & 23 & 13.7174 & 2.2293 \\
\hline
\end{tabular}

[Table/Fig-2]: The mean hepatic stiffness measured by ElastPQ PSWE for each

\begin{tabular}{|l|c|c|}
\hline & Correlation coefficient $(r)$ & Significance level $(p)$ \\
\hline Elast PQ SWE & 0.912 & $<0.0001$ \\
\hline APRI & 0.481 & $<0.0001$ \\
\hline FIB4 & 0.462 & $<0.0001$ \\
\hline
\end{tabular}

Diagnostic ability of ElastPQ PSWE to differentiate fibrosis stage: The optimal cut-off values of ElastPQ PSWE for different levels of fibrosis are listed with observed AUROCs and respective sensitivities, specificities and 95\% confidence intervals in [Table/Fig-4]. ElastPQ PSWE exhibited good diagnostic accuracy in identifying each liver fibrosis stage.

AUROC value of ElastPQ PSWE for predicting Mild/No fibrosis (FOF1), Significant fibrosis (F2), advanced fibrosis (F3) and cirrhosis (F4) was significantly higher than AUROC value of APRI or FIB4 [Table/Fig-5,6a-d].

\section{DISCUSSION}

In the present study, the diagnostic performance of ElastPQ PSWE for assessing hepatic fibrosis was analysed in 73 patients with hepatitis $B$ and $C$ infections of which ulltrasound image of two cases are shown in [Table/Fig-7,8]. The mean BMl was $25.51 \pm 3.28 \mathrm{~kg} / \mathrm{m}^{2}$ in present study which further confirms the notion that liver stiffness measurements obtained by ElastPQ PSWE are not influenced by $\mathrm{BMl}$, age and gender [15]. This supports the suggestion of Ferraioli $G$ et al., who recommended the use of ElastPQ PSWE technique as an ideal method for the non-invasive assessment of liver stiffness in patients with $\mathrm{BMl}>30 \mathrm{~kg} / \mathrm{m}^{2}$ [10].

In present study, there was significant positive correlation between different stages of liver fibrosis determined by liver biopsy and liver stiffness detected by ElastPQ PSWE ( $r=0.912$, $p<0.0001)$. Similar results were obtained in a study by Foucher $\mathrm{J}$ et al., [16]. Liver stiffness was significantly different between patients according to their fibrosis stage $(p<0.001)$ and significantly correlated with fibrosis stage $(r=0.73$, $p<0.0001$ ) [16]. Similarly, significant positive correlation between liver elastography and liver biopsy results $(r=0.664, p<0.001)$ in chronic hepatitis patients studied by Meng F et al., [17]

The diagnostic performance of ElastPQ PSWE for assessing no/early fibrosis (FO-F1), significant fibrosis (F2), severe fibrosis (F3), and liver cirrhosis (F4) was significantly high. The present study, to the best of our knowledge is one of the few studies to evaluate the diagnostic performance of ElastPQ PSWE in assessing hepatic fibrosis in patients with hepatitis $B$ and hepatitis $C$, considering liver biopsy as gold standard and providing a comparison with APRI and FIB4. 


\begin{tabular}{|l|c|c|c|c|c|c|c|c|c|c|}
\hline Stages & AUROC & $95 \% \mathrm{Cl}$ & Cut-off (kPa) & Sensitivity & Specificity & LR+ & LR- & PPV & NPV & p-value \\
\hline Mild/No fibrosis (FO-F1) & 0.980 & 0.916 to 0.999 & $\leq 3.9$ & 75.00 & 100 & 24.37 & 0.26 & 94.2 & 85.3 & $<0.0001$ \\
\hline Significant fibrosis (F2) & 0.840 & 0.735 to 0.915 & $\leq 7.2$ & 95.00 & 84.91 & 6.29 & 0.059 & 41.2 & 99.3 & $<0.0001$ \\
\hline Advanced fibrosis (F3) & 0.564 & 0.443 to 0.680 & $>7.2$ & 100.0 & 52.94 & 2.12 & 0.00 & 47.8 & 100.0 & 0.3464 \\
\hline Cirrhosis (F4) & 0.967 & 0.897 to 0.995 & $>10$ & 82.61 & 100.00 & -- & 0.17 & 100.0 & 92.6 & $<0.0001$ \\
\hline
\end{tabular}

[Table/Fig-4]: AUROC curve analysis for Elast PQ SWE for different stages of fibrosis.

AUROC: Area under the receiver operating characteristic curve; LR: Likelihood ratio test; PPV: Positive predictive value; NPV: Negative predictive value; $p \leq 0.0001$ Strongly significant

\begin{tabular}{|l|c|c|c|}
\hline Stages & $\begin{array}{c}\text { Elast PQ SWE } \\
\text { AUROC }(95 \% \mathrm{Cl})\end{array}$ & $\begin{array}{c}\text { APRI AUROC } \\
(95 \% \mathrm{Cl})\end{array}$ & $\begin{array}{c}\text { FIB4 AUROC } \\
(95 \% \mathrm{CI})\end{array}$ \\
\hline $\begin{array}{l}\text { Mild/No } \\
\text { fibrosis (F0-F1) }\end{array}$ & $0.980(0.916-0.990)$ & $0.730(0.619-0832)$ & $0.710(0.590-0.810)$ \\
\hline $\begin{array}{l}\text { Significant } \\
\text { fibrosis (F2) }\end{array}$ & $0.840(0.735-0.915)$ & $0.662(0.542-0.769)$ & $0.669(0.549-0.775)$ \\
\hline $\begin{array}{l}\text { Advanced } \\
\text { fibrosis (F3) }\end{array}$ & $0.564(0.443-0.680)$ & $0.528(0.408-0.646)$ & $0.500(0.381-0.620)$ \\
\hline $\begin{array}{l}\text { Cirrhosis (F4) } \\
\text { [Table/Fig-5]: Comparison of AUROC curve analysis. } \\
\text { AUROC: Area under the receiver operating characteristic curve }\end{array}$ & $0.751(0.636-0.845)$ \\
\hline
\end{tabular}
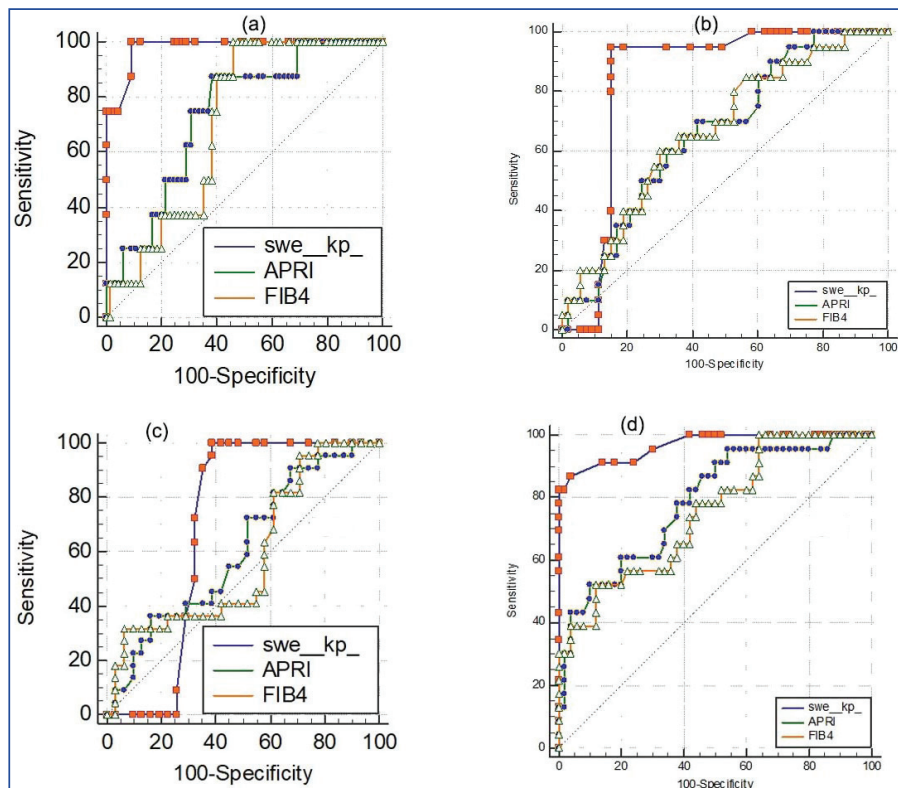

[Table/Fig-6]: a) AUROC curve for predicting mild/no fibrosis (FO-F1); b) AUROC curve for predicting significant fibrosis (F2); c) AUROC curve for predicting advanced fibrosis (F3); d) AUROC curve for predicting cirrhosis (F4).

SWE: Shear wave elastography; APRI: Aspartate to platelet ratio index; FIB4: Fibrosis 4 score
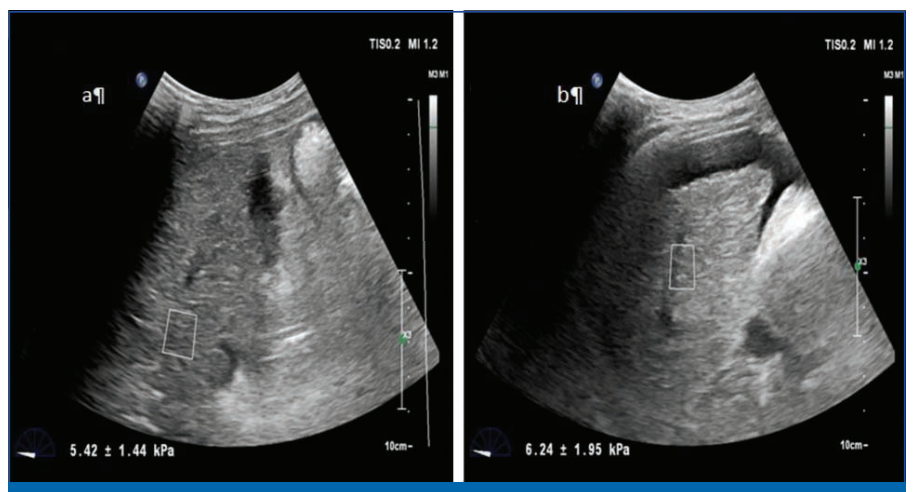

[Table/Fig-7]: Ultrasound Elastography images of a 62-year-old female, Hepatitis B and Hepatitis C positive, AST - 40U/L, ALT -35U/L, median elastography values were elevated $(6.2 \mathrm{kPa})$ corresponding to significant fibrosis which was confirmed by liver biopsy; a,b shows elastography values determined by Elast PQ point Shear Wave Elastography (SWE).

Present study results were in agreement with previous studies which evaluated SWE in liver fibrosis [Table/Fig-9] [10,16,18,19]. Foucher $\mathrm{J}$ et al., showed similar results with AUROC of 0.80 (0.75-0.84) for patients with significant fibrosis (F2) and 0.96 (0.94-0.98) for patients with cirrhosis (F4) [16]. Ferraioli $\mathrm{G}$ et al., reported AUROC of $0.80(0.75-0.84)$ for patients with significant fibrosis (F2) and 0.95 (0.86-0.93) for patients with cirrhosis (F4) [10]. While Ma JJ et al.,
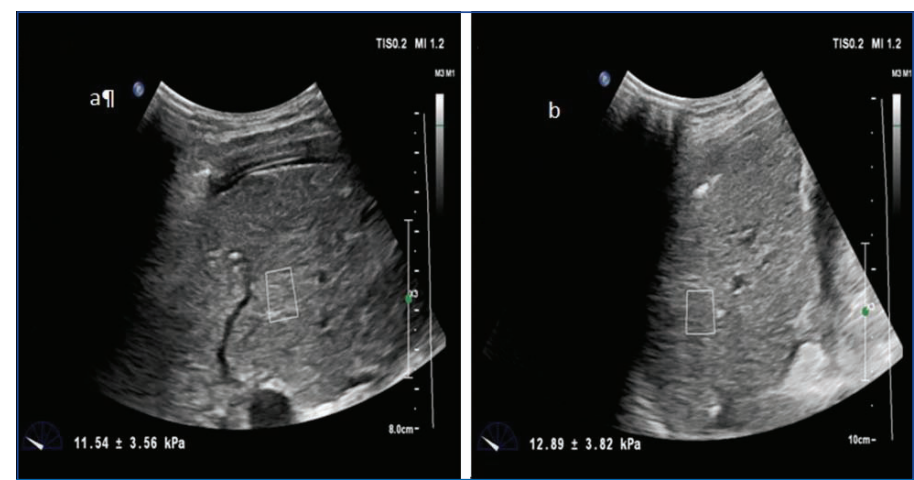

[Table/Fig-8]: Ultrasound Elastography images of a 43-year-old male, known case of hepatitis $B$ and, AST-37 U/L, ALT -41U/L, median elastography values were elevated $(12.8 \mathrm{kPa})$ corresponding to cirrhosis which was confirmed by liver biopsy. a,b) Shows elastography values determined by Elast PQ point Shear Wave Elastography (SWE).

reported AUROC of $0.94(0.75-0.84)$ for patients with significant fibrosis (F2), $0.90(0.86-0.93)$ for patients with severe fibrosis (F3), and 0.89 (0.94-0.98) for patients with cirrhosis (F4) [18]. Lee JE et al., reported AUROC of 0.86 (0.75-0.84) for patients with significant fibrosis (F2), $0.90(0.86-0.93)$ for patients with severe fibrosis (F3), and 0.855 (0.94-0.98) for patients with cirrhosis (F4) [19]. High diagnostic performance of Elast PQ PSWE helps in selecting appropriate antiviral treatment in viral hepatitis and for knowing treatment efficacy [6].

\begin{tabular}{|l|c|c|}
\hline Studies & Significant fibrosis (F2) & Cirrhosis (F4) \\
\hline Present study & 0.840 & 0.967 \\
\hline Foucher J et al., [16] & 0.80 & 0.96 \\
\hline Ferraioli G et al., [10] & 0.80 & 0.95 \\
\hline Ma JJ et al., [18] & 0.94 & 0.89 \\
\hline Lee JE et al., [19] & 0.86 & 0.85 \\
\hline
\end{tabular}

[Table/Fig-9]: AUROC liver elastography values in significant fibrosis (F2) and Cirrhosis (F4) in hepatitis patients in various studies $[10,16,18,19]$.

In present study, ElastPQ PSWE showed high NPV and specificity in staging liver fibrosis in hepatitis B and $\mathrm{C}$ patients for mild fibrosis/ significant fibrosis/advanced fibrosis and cirrhosis. Hence, ElastPQ PSWE can be considered as a screening test and can be useful in daily practice.

In this study, authors have compared the diagnostic performances of ElastPQ PSWE, APRI and FIB4 for liver fibrosis staging. ElastPQ PSWE exhibited higher diagnostic accuracy than the APRI and FIB4.

On review of literature, there were limited previous studies comparing the ElastPQ PSWE measurements and serum fibrosis indices. Lee JE et al., and Lu $Q$ et al., showed similar findings and concluded that liver stiffness (based on ElastPQ PSWE) demonstrated a significantly stronger correlation compared with fibrosis stages than APRI and FIB4 [19,20]. Similar findings were reported in the study of Castera $L$ et al., in patients with chronic hepatitis C [21].

Another important point for every newly developed technique is to establish the cut-off values that should be used to differentiate between stages of fibrosis. In present study, author used AUROC curve to know the optimal cut-off values of liver stiffness measurements by ElastPQ PSWE to differentiate different stages of fibrosis. The optimum cutoff values for stage FO-F1, F2, F3, F4 patients with chronic hepatitis $\mathrm{B}$ and $\mathrm{C}$ is $\leq 3.9 \mathrm{kPa}, \leq 7.2 \mathrm{kPa}, \geq 7.2 \mathrm{kPa}$ and $\geq 10 \mathrm{kPa}$. Ma JJ et al., 
offered the optimal cut-off values for predicting significant fibrosis $(<\mathrm{F} 2,6.99 \mathrm{kPa})$ and cirrhosis (F4, $9.19 \mathrm{kPa})$, which were comparable to present study [18]. Ferraioli $\mathrm{G}$ et al., defined higher cut-off values 7.3 and $13.3 \mathrm{kPa}$ for predicting significant fibrosis (F2) and cirrhosis (F4), respectively in chronic hepatitis C patients [10].

\section{Limitation(s)}

Firstly, the sample size was small, however when non-invasive tests are in vogue for detecting fibrosis and liver biopsy in itself associated with limitations and complications considering a large sample is cumbersome.

Secondly, author used liver biopsy as gold standard which can show sampling variability and there can be a lack of precise correlation between site of ultrasound liver stiffness measurements and site of tissue biopsy which can influence on study results. These variability's further highlights the deficiencies of liver biopsy as an imperfect gold standard. Though in present study, author have done ElastPQ PSWE measurements and biopsy in similar anatomic region.

Comparison with other non-invasive elastographic techniques such as transient elastography was not done in which literature is in substantial amount owing to longer period of time the technique is available. However, ElastPQ PSWE has advantages over transient elastography that it can use in patients with ascites and patients with high BMI [7]. Also, author did not study other morbid conditions that might affect the liver visco-elasticity such as iron overload.

\section{CONCLUSION(S)}

ElastPQ PSWE is a promising non-invasive method for the evaluation of liver fibrosis, with high diagnostic performance and high NPV and high specificity which can be used in day to day practice for treatment and follow-up of patients with hepatitis B and C infection. Since, ElastPQ PSWE technique is integrated into USG machines it can easily be available, economically feasible alternative to liver biopsy in patients with hepatitis B and C infection for treatment and follow-up which can make an important impact medically.

\section{REFERENCES}

[1] Mobarak L, Nabeel MM, Hassan E, Omran D, Zakaria Z. Real-time elastography as a noninvasive assessment of liver fibrosis in chronic hepatitis C Egyptian patients: A prospective study. Ann Gastroenterol. 2016;29:358-62.

[2] Myers RP, Elkashab M, Ma M, Crotty P, Pomier-Layrargues G. Transient elastography for the noninvasive assessment of liver fibrosis: A multicentre Canadian study. Can J Gastroenterol. 2010;24(11):661-70.

[3] European Association for the Study of the Liver. EASL clinical practice guidelines: Management of hepatitis C virus infection. J Hepatol. 2011;55:245-64.
[4] Bedossa P, Dargère D, Paradis V. Sampling variability of liver fibrosis in chronic hepatitis C. Hepatology. 2003;38:1449-57.

[5] Manning DS, Afdhal NH. Diagnosis and quantitation of fibrosis. Gastroenterology. 2008;134:1670-81.

[6] Lee JE, Lee JM, Lee KB, Yoon JH, Shin Cl, Han JK, et al. Non invasive assessment of hepatic fibrosis in patients with chronic hepatitis $B$ viral infection using magnetic resonance elastography. Korean J Radiol. 2014;15:210-17.

[7] Barr RG, Ferraioli G, Palmeri ML, Goodman ZD, Garcia-Tsao G, Rubin J, et al. Elastography assessment of liver fibrosis: society of radiologists in ultrasound consensus conference statement. Radiology. 2015;276:845-61.

[8] Ferraioli G, Parekh P, Levitov AP, Filice C. Shear wave elastography for evaluation of liver fibrosis. J Ultrasound Med. 2014;33:197-203.

[9] Sporea I, Bota S, Gradinaru-Tas cau O, Sirli R, Popescu A. Comparative study between two point shear wave elastographic techniques: Acoustic Radiation Force Impulse (ARFI) elastography and ElastPQ. Med Ultrason. 2014;16(4):309-14.

[10] Ferraioli G, Tinelli C, Lissandrin R, Zicchetti M, Bello BD, Filice G, et al. Reproducibility and performance of a new point shear wave elastography technique for assessing fibrosis in chronic Hepatitis C. World J Gastroenterol. 2014;20(16):4787-96.

[11] Castéra L, Foucher J, Bernard P-H, Carvalho F, Allaix D, Merrouche W, et al. Pitfalls of liver stiffness measurement: A 5-year prospective study of 13,369 examinations. Hepatology. 2010;51:828-35.

[12] Giannini E, Risso D, Botta F, Chiarbonello B, Fasoli A, Malfatti F, et al. Validity and clinical utility of the aspartate aminotransferase alanine aminotransferase ratio in assessing disease severity and prognosis in patients with hepatitis $\mathrm{C}$ related chronic liver disease. Arch Intern Med. 2003;163:218-24.

[13] Wai C, Greenson JK, Fontana RJ, Kalbfleisch JD, Marrero JA, Conjeevaram HS et al. A simple noninvasive index can predict both significant fibrosis and cirrhosis in patients with chronic hepatitis C. Hepatology. 2003;38:518-26.

[14] Bedossa P, Poynard T. The METAVIR Cooperative Study Group. An algorithm for the grading of activity in chronic hepatitis C. Hepatology. 1996;24:289-93.

[15] Fraquelli M, Baccarin A, Casazza G, Conti CB, Giunta M, Massironi S, et al. Liver stiffness measurement reliability and main determinants of point shearwave elastography in patients with chronic liver disease. Aliment Pharmacol Ther. 2016:44(4):356-65.

[16] Foucher J, Chanteloup E, Vergniol J, Castéra L, Le Bail B, Adhoute X, Bertet J, Couzigou P, et al. (FibroScan): Diagnosis of cirrhosis by transient elastography (FibroScan): A prospective study. Gut. 2006;55:403-08

[17] Meng F, Zheng Y, Zhang Q, Mu Z, Xu X, Zhang H, et al. Noninvasive evaluation of liver fibrosis using real-time tissue elastography and transient elastography (FibroScan). J Ultrasound Med. 2015;34:403-10.

[18] Ma JJ, Ding H, Mao F, Sun HC, Xu C, Wang WP. Assessment of liver fibrosis with elastography point quantification technique in chronic hepatitis B virus patients: A comparison with liver pathological results. J Gastroenterol Hepatol. 2014;29:814-19.

[19] Lee JE, Shin KS, Cho JS, You SK, Min JH, Kim KH, et al. Noninvasive assessment of liver fibrosis with ElastPQ: Comparison with transient elastography and serologic fibrosis marker tests, and correlation with liver pathology results. Ultrasound Med Biol. 2017;43(11):2515-21.

[20] Lu Q, Lu C, Li J, Ling W, Qi X, He D, et al. Stiffness value and serum biomarkers in liver fibrosis staging: Study in large surgical specimens in patients with chronic hepatitis B. Radiology. 2016;280(1):290-99.

[21] Castera L, Winnock M, Pambrun E, Paradis V, Perez P, Loko MA, et al. Comparison of transient elastography (FibroScan), FibroTest, APRI and two algorithms combining these noninvasive tests for liver fibrosis staging in HIV/ HCV coinfected patients: ANRS CO13 HEPAVIH and FIBROSTIC collaboration. HIV Med. 2014;15(1):30-39.

\section{PARTICULARS OF CONTRIBUTORS:}

1. Associate Professor, Department of Radiology, Mysore Medical College and Research Institute, Mysore, Karnataka, India.

2. Senior Resident, Department of Radiology, Mysore Medical College and Research Institute, Mysore, Karnataka, India.

3. Senior Resident, Department of Radiology, Mysore Medical College and Research Institute, Mysore, Karnataka, India.

4. Assistant Professor, Department of Radiology, Mysore Medical College and Research Institute, Mysore, Karnataka, India.

5. Professor, Department of Radiology, Mysore Medical College and Research Institute, Mysore, Karnataka, India.

6. Senior Resident, Department of Radiology, Mysore Medical College and Research Institute, Mysore, Karnataka, India.

7. Senior Resident, Department of Radiology, Mysore Medical College and Research Institute, Mysore, Karnataka, India.

8. Senior Resident, Department of Radiology, Mysore Medical College and Research Institute, Mysore, Karnataka, India.

NAME, ADDRESS, E-MAIL ID OF THE CORRESPONDING AUTHOR:

Dr. Nagarajaiah Chandandur Pradeepkumar,

No. 371 ,Samhita, $2^{\text {nd }}$ Main, $3^{\text {rd }}$ Cross, Behind Bharath Petrolbunk, Niveditha Nagar, Mysore-570022, Karnataka, India.

E-mail: radmmc@gmail.com

\section{AUTHOR DECLARATION:}

- Financial or Other Competing Interests: None

- Was Ethics Committee Approval obtained for this study? Yes

- Was informed consent obtained from the subjects involved in the study? Yes

- For any images presented appropriate consent has been obtained from the subjects. Yes
PLAGIARISM CHECKING METHODS: JJain Het al.]

- Plagiarism X-checker: Nov 12, 2020

- Manual Googling: Dec 18, 2020

- iThenticate Software: Jan 28, 2021 (24\%)
ETYMOLOGY: Author Origin

Date of Peer Review: Nov 26, 2020

Date of Acceptance: Dec 21, 2020

Date of Online Ahead of Print: Jan 29, 2021

Date of Publishing: Apr 01, 2021 